\title{
Transferência de calor e massa: Fusão de uma placa de gelo
}

\author{
Heat and mass transfer: Melting of an ice sheet
}

\author{
R. L. Garcia ${ }^{1}$, J. Zabadal ${ }^{2}$, R. A. Amaral ${ }^{* 2}$, J. A. D. G. Neto ${ }^{3}$, A. Schmitz ${ }^{1}$ \\ ${ }^{1}$ Universidade Estadual do Rio Grande do Sul, Novo Hamburgo, RS, Brasil \\ ${ }^{2}$ Departamento Interdisciplinar, Universidade Federal do Rio Grande do Sul, Tramandaí, RS, Brasil \\ ${ }^{3}$ Departamento de Biologia Molecular e Biotecnologia, Instituto de Biociências, Centro de Biotecnologia, \\ Universidade Federal do Rio Grande do Sul, Porto Alegre, RS, Brasil
}

Recebido em 22 de Novembro de 2016. Revisado em 27 de Dezembro de 2016. Aceito em 29 de Dezembro de 2016

Esse trabalho descreve um experimento simples e de fácil execução, destinado ao estudo da transferência de calor e massa por meio de uma aula prática. Tal estudo é realizado a partir da fusão de uma placa de gelo, ao ar ambiente, e com base na avaliação quantitativa do calor transferido por radiação, convecção e condensação de vapor d'água sobre a superfície dessa placa. Os valores teóricos, previstos a partir de equações relativas a esses processos de transferência de calor e massa, são comparados com dados experimentais relativos à taxa mássica de fusão dessa placa. A facilidade na execução dos ensaios e a simplicidade do equipamento empregado torna esse experimento uma atividade prática de fácil implementação em disciplinas que tratam da transferência de calor e massa. Além disso, a concordância entre valores teórico e experimental da taxa de transferência de calor e massa, e a metodologia empregada na análise e quantificação dessa taxa, viabilizam o experimento proposto como uma ferramenta pedagógica eficiente para o estudo desse tema.

Palavras-chave: transferência de calor e massa, fusão de uma placa de gelo, radiação, convecção, condensação de vapor d'água.

This work describes a simple and easy-to-perform experiment, destined to the study of the heat and mass transfer by means of a practical class. This study is carried out from the melting of an ice sheet into ambient air and based on the quantitative evaluation of the heat transferred by radiation, convection and condensation of water vapor on the surface of that plate. The theoretical values, predicted from equations related to these processes of heat and mass transfers, are compared with experimental data concerning the melting mass rate of this plate. The ease of running the tests and the simplicity of the equipment employed makes this experiment a practical activity that is easy to implement in disciplines dealing with heat and mass transfer. In addition, the agreement between theoretical and experimental values of the heat and mass transfer rate, and the methodology used in the analysis and quantification of this rate, make feasible the proposed experiment as an efficient pedagogical tool to study this subject. Keywords: heat and mass transfer, melting of an ice sheet, radiation, convection, condensation of water vapor.

\section{Introdução}

Em muitas disciplinas dos cursos de Engenharia, o conteúdo é ministrado através de aulas teóricoexpositivas, nas quais são apresentadas as equações que regem os processos físicos objeto de estudo, complementadas pela resolução de exercícios numéricos que simulam situações reais. Na área de Fenômenos

*Endereço de correspondência: rafael.amaral@ufrgs.br. de Transporte, uma série de fatores pode tornar essa metodologia de ensino ineficiente, entre os quais destacamos:

1. a extensão do conteúdo programático;

2. a grande quantidade de equações, bem como a série de formulações analíticas utilizadas na dedução dessas equações; 
3. a interpretação falha ou incorreta, por parte dos alunos, do significado de algumas grandezas físicas.

Cabe destacar que as disciplinas de Fenômenos de Transporte são fundamentais na formação de um engenheiro, pois sem a clara compreensão dos processos físicos ali tratados, o aluno não estará apto ao aprendizado das Operações Unitárias, do Cálculo de Reatores, de Trocadores de Calor e outras disciplinas aplicadas a processos industriais [1].

As dificuldades inerentes ao ensino dos Fenômenos de Transporte podem ser contornadas a partir da realização de aulas práticas, que tendem a propiciar aos alunos uma compreensão mais clara dos fenômenos físicos presentes num sistema real, a partir da avaliação das alterações das propriedades macroscópicas desse sistema e das grandezas físicas associadas $[2,3]$. Contudo, a realização de aulas práticas é, muitas vezes, obstaculizada por entraves financeiros e burocráticos na aquisição dos equipamentos necessários à execução de experimentos, resultando na ausência (ou falta) desse tipo de atividade em muitas instituições de ensino [4]. O emprego de equipamentos simples, de baixo custo e confeccionados de forma a viabilizar ensaios de fácil execução, possibilita a inclusão desse recurso didático para o ensino de Fenômenos de Transporte [5].

Nesse contexto, foi elaborado um experimento destinado ao estudo da transferência de calor e massa, no qual uma placa de gelo posicionada verticalmente sofre processo de fusão por exposição ao ar ambiente. A simplicidade do aparato empregado para a realização dos ensaios e a facilidade na obtenção dos dados experimentais relativos a taxa de transferência de calor e massa tornam o experimento descrito uma alternativa interessante para docentes que buscam incluir aulas práticas em disciplinas de Fenômenos de Transporte.

\section{Fundamentos teóricos}

O estudo da transferência de calor é um dos temas mais importantes num curso de Engenharia, e a necessidade de avaliar, quantificar e controlar esse processo abrange a grande maioria dos sistemas e equipamentos. Admite-se que o calor é transferido de três modos (ou mecanismos) distintos, cuja caracterização é feita a partir de modelos baseados nas leis de Fourier, de resfriamento de Newton e de StefanBoltzmann; que tratam da condução, convecção e radiação, respectivamente. Cabe aqui ressaltar que, em sistemas físicos reais, os três mecanismos de transferência de calor estão presentes; e, consequentemente, o calor transferido é resultado da soma das contribuições correspondentes a cada um deles [6]. Em algumas situações práticas, a predominância de um dos mecanismos permite desprezar a contribuição dos outros dois modos de transferência de calor; contudo, a adoção dessa hipótese deve ser feita de forma criteriosa, sob pena de se obter valores, a partir das correlações teóricas, que se afastam em demasia da situação real. O equacionamento a seguir é baseado em referências clássicas do conteúdo de fenômenos de transporte (transferência de calor, massa e momento) [6-12].

\subsection{Convecção do calor}

A lei de resfriamento de Newton nos diz que a taxa de transferência de calor $q$ numa interface sólida é diretamente proporcional ao produto da área $A$ dessa interface pela diferença de temperatura entre o fluido e a parede do sólido nele imerso, sendo o coeficiente de película $h$ a constante de proporcionalidade, conforme explicitado na equação (1):

$$
q=h A\left(\mathrm{~T}_{\mathrm{s}}-\mathrm{T}_{\infty}\right)
$$

Nessa equação as temperaturas da superfície do sólido e do fluido circundante são admitidas como sendo constantes e iguais a $T_{s}$ e $T_{\infty}$, respectivamente. A simplicidade da formulação analítica da Equação (1) oculta dificuldades na obtenção de valores precisos para o coeficiente de película, tendo em vista que esse coeficiente está associado à geometria da superfície, bem como às condições de escoamento e às propriedades do fluido que escoa na interface sólida. A multiplicidade de variáveis independentes associadas ao valor de $\mathrm{h}$ conduz à adoção de uma série de hipóteses simplificativas, sendo possível encontrar na literatura uma enorme gama de correlações empíricas para esse coeficiente, aplicadas a situações específicas [7]. Na convecção natural de um gás numa interface sólida, os valores do coeficiente de película situam-se na faixa de 5 a $25 \mathrm{~W} /\left(\mathrm{m}^{2} \mathrm{~K}\right)$ [8]. Para a convecção natural em uma placa vertical com $0,3 \mathrm{~m}$ de altura e submetida a uma diferença de temperatura de $30^{\circ} \mathrm{C}$, é estimado um coeficiente de película de $4,5 \mathrm{~W} /\left(\mathrm{m}^{2} \mathrm{~K}\right)$ [9].

No experimento proposto, o ar ambiente que entra em contato com a superfície sólida (placa de gelo) irá se resfriar e, como consequência do aumento de 
sua densidade, será induzido um fluxo descendente desse fluido. Dessa forma, o escoamento decorre de um processo de convecção natural, provocado pelas forças de empuxo que se originam das diferenças de densidade devidas às variações de temperatura do ar. Haverá, então, sobre a superfície da placa, uma região caracterizada por um gradiente de temperatura e onde irá ocorrer a transferência de calor, denominada camada limite térmica [10]. As propriedades do filme de ar nessa região são estimadas numa temperatura que corresponde à média entre a superfície sólida, $T_{s}$, e a do fluido circundante, $T_{\infty}$.

Uma das formulações utilizadas para o cálculo do coeficiente de película em um processo de convecção natural é baseada no número de Grashoff $\left(N_{g r}\right)$, apresentado na equação (2):

$$
N_{g r}=\frac{g \beta\left(T_{s}-T_{\infty}\right) L^{3}}{\gamma^{2}}
$$

na qual $g, L, \beta, \alpha$ e $\gamma$ correspondem, respectivamente, à aceleração da gravidade, à altura da placa, ao coeficiente de expansão térmica, à difusividade térmica e à viscosidade cinemática do ar na temperatura do filme. Correlações envolvendo os números de Nusselt $\left(N_{n u}\right)$ e Prandtl $\left(N_{p r}\right)$ na convecção natural sobre uma placa vertical são dadas pelas equações (3), (4) e (5):

$$
\begin{gathered}
N_{n u}=0,59(G r P r)^{0,25} \\
N_{n u}=\frac{h L}{k} \\
N_{p r}=\frac{\gamma}{\alpha}
\end{gathered}
$$

sendo $k$ a condutividade térmica do ar na temperatura de filme [11].

\subsection{Calor transferido por condução}

$\mathrm{Na}$ transferência de calor entre um fluido e uma superfície sólida, a movimentação das partículas desse fluido é consequência de um movimento aleatório (difusão) a nível molecular, o qual é típico da condução do calor, e de um movimento macroscópico, sendo ambos os processos abrangidos pelo mecanismo da convecção [12]. Assim, uma eventual contribuição do mecanismo de condução na taxa global de transferência de calor está descartada a priori.

\subsection{Calor transferido por radiação}

Resta estimar o calor transferido por radiação, quantificado pela Lei de Stefan-Boltzmann, dada pela equação (6):

$$
q_{\text {rad }}=\varepsilon \sigma A\left(T_{\infty}^{4}-T_{s}^{4}\right)
$$

na qual $\sigma$ é a constante de Stefan-Boltzmann e $\varepsilon$ é a emissividade da superfície sólida, sendo que os valores desse coeficiente para o gelo situam-se na faixa de 0,95 a 0,98 na temperatura de $0{ }^{\circ} \mathrm{C}$ [13]. A presença de uma lâmina de água de pequena espessura sobre a placa não altera a emissividade da superfície, tendo em vista que o valor de $\varepsilon$ para a água é de 0,96 [13].

\subsection{Transferência simultânea de calor e massa}

Ao se resfriar, uma parcela do vapor de água contido no ar poderá se condensar sobre a superfície da placa. O calor liberado na condensação é absorvido na superfície da placa numa taxa que é dada pela equação (7):

$$
q_{m}=\dot{m}_{\text {cond. }} L_{\text {vap }}
$$

na qual os termos e $\dot{m}_{\text {cond. }}$ e $L_{\text {vap. }}$ correspondem, respectivamente, à taxa de condensação do vapor d'água sobre a superfície da placa e ao calor de vaporização da água. A condensação irá ocorrer numa taxa proporcional à diferença de concentração do vapor d'água junto à superfície da placa, $\rho_{v, s}$, e no fluido circundante, $\rho_{v, \infty}$, conforme a equação (8):

$$
\dot{m}_{\text {cond. }}=h_{m} A\left(\rho_{\mathrm{v}, \mathrm{s}}-\rho_{\mathrm{v}, \infty}\right)
$$

sendo $h_{m}$ o coeficiente de transferência convectiva de massa. A similaridade entre as equações (1) e (8) resulta do fato de que há uma analogia entre os processos de transferência de calor e massa. O cálculo do coeficiente $\mathrm{h}_{\mathrm{m}}$ envolve o número de Sherwood $\left(N_{s h}\right)$ e o número de Schmidt $\left(N_{s c}\right)$, segundo as equações (9), (10) e (11):

$$
\begin{gathered}
N_{s h}=0,59(G r S c)^{0,25} \\
N_{s h}=\frac{h_{m} L}{D_{A B}} \\
N_{s c}=\frac{\gamma}{D_{A B}}
\end{gathered}
$$

que apresentam similaridade com as equações (3) a (5). A difusividade mássica da substância A através 
da substância B (no caso, vapor d'água no ar), $D_{A B}$, é uma propriedade física com a mesma dimensão da difusividade térmica $(\alpha)$ [14], sendo necessário atentar para a expressão do número de Grashoff $\left(N_{g r}\right)$ vinculado à transferência de massa, conforme equação 12):

$$
N_{g r}=\frac{g\left(\rho_{s}-\rho_{\infty}\right) L^{3}}{\rho \gamma^{2}}
$$

na qual os termos $\rho, \rho_{s}$ e $\rho_{\infty}$ correspondem à concentração mássica do ar na temperatura de filme, junto à interface sólida e na corrente livre, respectivamente. Os valores de concentração mássica do ar e do vapor d 'água são calculados com base na hipótese de que essas substâncias possuem comportamento próximo ao do gás ideal, e admitindo que o ar é o resultado da mistura de ar seco com vapor d'água, na pressão de $1 \mathrm{~atm}$. A Tabela 1 apresenta um quadro com as expressões matemáticas empregadas nos cálculos de concentração mássica.

Cabe destacar que a taxa de condensação do vapor d'água, dada pela equação (8), é proporcional à diferença de concentração mássica do vapor d'água na superfície da placa e na corrente livre; sendo que, na corrente livre, a pressão de vapor d'água é proporcional à umidade relativa do ar (UR). O número de Grashoff, dado pela equação (12), é proporcional à diferença de concentração mássica do ar na superfície da placa e na corrente livre.

\section{Execução dos ensaios}

O experimento proposto fundamenta-se na estimativa da taxa de transferência de calor convectivo, entre uma superfície sólida e o fluido circundante, a partir da massa de sólido que é derretida como consequência do calor absorvido na superfície e da massa de água transferida do ar circundante.

Utilizando uma fôrma metálica, obtém-se uma placa de gelo que é suspensa verticalmente e cujo formato - área frontal significativamente maior que a soma das áreas laterais - tende a produzir um escoamento descendente de ar, promovido pelas forças de empuxo que governam a convecção natural do fluido.

Em consequência do derretimento contínuo dessa placa, haverá a presença de uma película de água que flui na interface sólida e produz gotejamento na extremidade inferior da placa, devendo o líquido assim produzido ser recolhido através de uma canaleta e conduzido para uma proveta. Durante o experimento, a cada minuto, devem ser feitas medições do volume de líquido escoado nesse intervalo.

$\mathrm{Na}$ realização dos ensaios, foi utilizada uma placa de gelo, na forma de um paralelepípedo, com dimensões de $285 \mathrm{~mm}$ x $185 \mathrm{~mm}$ x $15 \mathrm{~mm}$, o que corresponde a uma área superficial de $0,120 \mathrm{~m}^{2}$. Uma estrutura confeccionada para manter a placa de gelo suspensa no ar e fixar uma canaleta destinada ao recolhimento da água derretida, também foi empregada nesse experimento, conforme ilustrado na Figura 1.

\section{Metodologia utilizada}

A transferência de calor entre o ar circundante e a superfície sólida resulta no derretimento do gelo. $\mathrm{O}$ produto da massa $m$ de água assim produzida pelo calor latente de fusão do gelo, $L_{f}$, corresponde ao calor absorvido na superfície; sendo possível estimar a taxa de calor transferido dada pela equação 113 :

$$
q=\frac{m L_{f}}{\Delta t}
$$

na qual $\Delta t$ é o intervalo de tempo necessário para a fusão de uma massa $m$ de gelo. A taxa calculada na equação (13), a partir dos registros das quantidades de gelo que derretem a cada intervalo de tempo, deve ser confrontada com a potência térmica resultante dos processos de convecção, radiação e transferência de massa, cuja formulação analítica foi explicitada na seção 2. Um balanço térmico na superfície da placa pode ser assim expresso na equação (14):

$h A\left(\mathrm{~T}_{\infty}-\mathrm{T}_{\mathrm{s}}\right)+\varepsilon \sigma A\left(T_{\infty}^{4}-T_{s}^{4}\right)+\dot{m}_{\text {cond. }} L_{v a p .}=q_{g}$

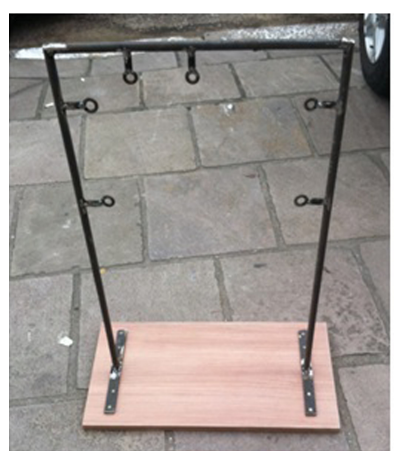

(a) Estrutura de fixação

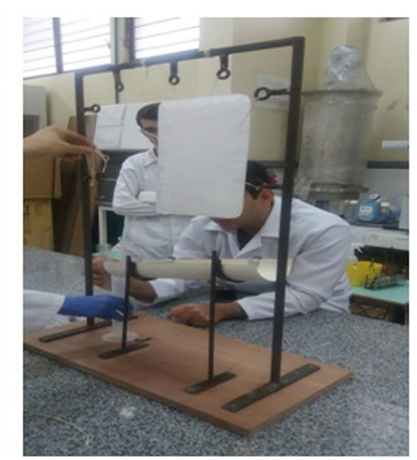

(b) Placa de gelo fixada
Figura 1: Imagens da estrutura utilizada para fixação da placa de gelo (esquerda) e de uma aula prática na qual é realizado o experimento proposto (direita). 
Tabela 1: Equações utilizadas no cálculo das concentrações mássicas.

\begin{tabular}{|c|c|c|c|}
\hline \multicolumn{2}{|c|}{ Superfície da placa $\left(t_{\mathrm{s}}, \mathrm{T}_{\mathrm{s}}\right)$} & \multicolumn{2}{|c|}{ Corrente livre $\left(\mathrm{t}_{\infty}, \mathrm{T}_{\infty}\right)$} \\
\hline pressão de & \multirow{3}{*}{$\frac{610,8}{101300} \exp \left(\frac{17,3 t}{t+237,3}\right)$} & pressão de & \multirow{3}{*}{$\frac{U R 610,8}{101300} \exp \left(\frac{17,3 t}{t+237,3}\right)$} \\
\hline vapor & & vapor & \\
\hline $\mathrm{p}_{\mathrm{v}, \mathrm{s}}(\mathrm{atm})$ & & $\mathrm{p}_{\mathrm{v}, \infty}(\mathrm{atm})$ & \\
\hline concentração & \multirow{3}{*}{$\frac{28,8}{0,082 T}$} & concentração & \multirow{3}{*}{$\frac{28,8}{0,082 T}$} \\
\hline mássica do ar seco & & mássica do ar seco & \\
\hline$\rho_{\mathrm{a}, \mathrm{s}}(\mathrm{g} / \mathrm{L})$ & & $\rho_{\mathrm{a}, \infty}(\mathrm{g} / \mathrm{L})$ & \\
\hline concentração & \multirow{3}{*}{$\frac{p_{v, s} 18}{0,082 T}$} & concentração & \multirow{3}{*}{$\frac{p_{v, \infty} 18}{0,082 T}$} \\
\hline $\begin{array}{l}\text { mássica do vapor } \\
\text { d’água }\end{array}$ & & $\begin{array}{l}\text { mássica do vapor } \\
\text { d'água }\end{array}$ & \\
\hline$\rho_{\mathrm{v}, \mathrm{s}} \quad(\mathrm{g} / \mathrm{L})$ & & $\rho_{\mathrm{v}, \infty} \quad(\mathrm{g} / \mathrm{L})$ & \\
\hline concentração & \multirow{2}{*}{$\rho_{s}=\rho_{a, s}+\rho_{v, s}$} & concentração & \multirow[t]{2}{*}{$\rho_{\infty}=\rho_{a, \infty}+\rho_{v, \infty}$} \\
\hline$\rho_{\mathrm{s}}(\mathrm{g} / \mathrm{L})$ & & $\rho_{\infty}(\mathrm{g} / \mathrm{L})$ & \\
\hline
\end{tabular}

sendo $q_{g}$ o valor correspondente à potência térmica total transferida para a placa, cujo efeito será o derretimento do gelo. Cabe aqui ressaltar que a quantidade de gelo derretida é inferior àquela mensurada no experimento, tende em vista que uma parcela da água recolhida na canaleta provem da condensação do vapor d'água sobre a superfície da placa, de modo que a equação (13) deve ser reescrita conforme apresentada na equação (15):

$$
q_{g}=\frac{m L_{f}}{\Delta t}-\dot{m}_{\text {cond. }} L_{f}
$$

Na Figura 2 é apresentado um esquema visando ilustrar os fenômenos envolvidos na transferência de calor entre o ar ambiente e a placa.

Em ensaio realizado durante aula prática, com temperatura do ar seco a $21^{\circ} \mathrm{C}$ e umidade relativa do ar de $81 \%$, foi registrada a massa de gelo fundida a cada minuto. Os dados experimentais assim obtidos foram plotados num gráfico, apresentado na Figura 3.

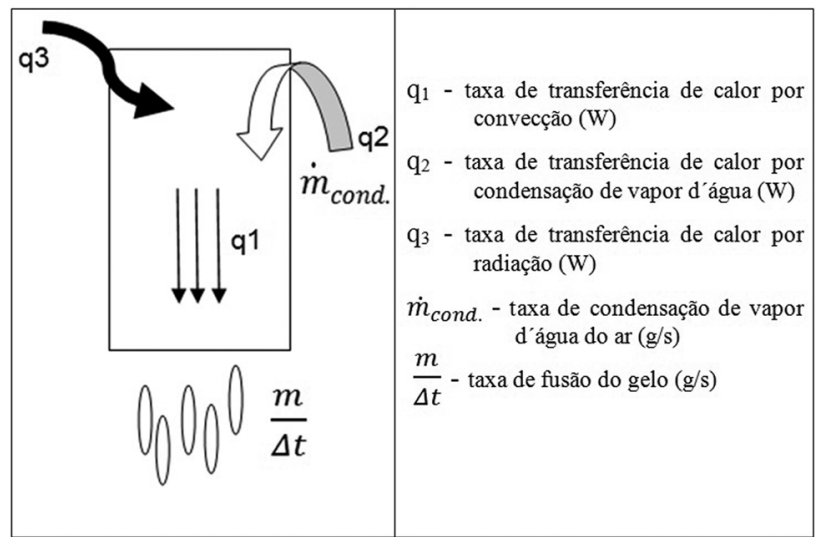

Figura 2: Imagem ilustrativa dos fenômenos envolvidos no ensaio realizado, detalhando as taxas de transferência de calor e de massa na placa de gelo.

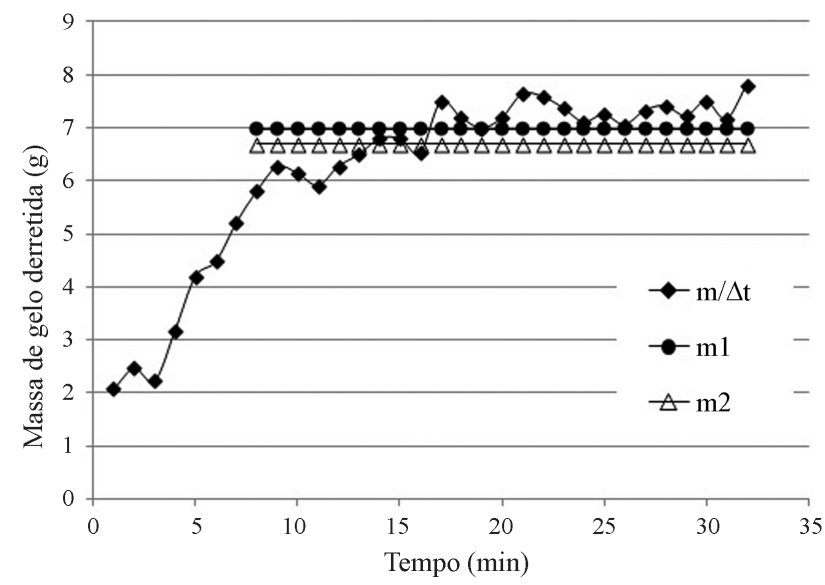

Figura 3: Gráfico representativo da variação da taxa aparente de fusão do gelo com o tempo. Os pontos circulares e triangulares correspendem, respectivamente, à taxa média aparente e real de fusão do gelo.

Nesse gráfico, o valor médio da taxa aparente de fusão do gelo, $m_{1}$, corresponde à massa média de água coletada por unidade de tempo, a partir de $\mathrm{t}=8$ min. A taxa real de fusão do gelo, $m_{2}$, equivale ao valor da taxa aparente descontada da taxa de condensação do vapor d'água, calculada na equação (8), tendo em vista que essa parcela de água não é proveniente da fusão da placa de gelo. Os valores de $\mathrm{m}_{1}$ e $\mathrm{m}_{2}$ foram inseridos nesse gráfico na forma de uma linha horizontal que se inicia a partir do oitavo intervalo de tempo; tendo em vista que, no início da contagem do tempo, a taxa de fusão do gelo era muito baixa, se estabilizando após alguns minutos.

Para a realização dos cálculos, adotou-se a temperatura de $0{ }^{\circ} \mathrm{C}$ para a superfície da placa e as propriedades termofísicas do ar foram avaliadas na 
temperatura de filme do ensaio: $10,5{ }^{\circ} \mathrm{C}$. A Tabela 2 detalha os resultados obtidos.

\section{Discussão dos resultados}

O desvio entre os valores teórico e experimental da taxa de transferência de calor é de $\mathbf{7 , 1 2} \%$. Tal discrepância é significativamente pequena, considerando o grau de incerteza associado aos parâmetros utilizados para caracterizar o sistema físico real e o fato de que são empregadas equações empíricas no cálculo do coeficiente de película e do coeficiente convectivo de transferência de massa. Diante disso, admite-se que a metodologia utilizada, descrita pelo balanço térmico da equação (14), representa adequadamente o comportamento do sistema objeto de análise.

A verificação de um processo real, no qual a transferência de calor ocorre por três mecanismos distintos, faz o aluno se deparar com uma situação diversa da metodologia classicamente empregada no estudo de Fenômenos de Transportes, apontada no início da seção 1. Em aulas teórico-expositivas, a sistematização empregada no estudo de modelos correspondentes aos três mecanismos fundamentais de transferência de calor, ainda que útil do ponto de vista didático, tende a induzir o aluno a selecionar um mecanismo de transferência de calor e uma equação específica para cada problema numérico proposto, fazendo com que ele possa a vir a enfrentar dificuldades na análise dos mecanismos de transferência que predominam numa situação real.

Nesse ponto cabe ressaltar que o experimento proposto consiste na verificação de um modelo de balanço térmico baseado em três mecanismos de transferência de calor, obtendo-se valores experimentais muito próximos aos valores teóricos previstos pelo modelo. Dessa forma, o experimento enquadrase de forma satisfatória como ferramenta pedagógica de verificação de leis e teorias e, simultaneamente, incentiva o aluno a refletir sobre os mecanismos e efeitos da transferência de calor ao tratar de uma situação real na qual todos os três mecanismos envolvidos são relevantes [15]. Nesse contexto, foi discutido com os alunos, durante a realização da aula prática, o fato de haver um crescimento contínuo da taxa de fusão do gelo no início do experimento, seguida da estabilização dessa taxa.

O comportamento térmico do sistema na fase inicial do experimento revela que o calor absor- vido inicialmente produz um aquecimento da placa, cuja temperatura média é significativamente menor que sua temperatura de fusão, pois o gelo está em equilíbrio térmico com a temperatura do freezer do qual foi retirado. Assim, no início do processo, há uma absorção de calor sensível na placa; e, após algum tempo, tendo em vista que sua espessura é proporcionalmente menor que a área, a placa pode ser considerada termicamente uniforme. Dessa forma, no cálculo da taxa aparente de fusão do gelo não foi computado o período de crescimento contínuo da taxa. Essa análise, embora realizada do ponto de vista qualitativo, trata-se de uma visão típica de engenharia, que pode ser concebida a partir do gráfico ilustrado na Figura 3, e leva o aluno a refletir sobre os efeitos da transferência de calor em um problema real.

A utilização de uma placa de gelo como superfície de troca térmica garante uma estabilidade na temperatura da interface sólida, diferentemente do que ocorre quando o objeto sólido absorve ou cede calor sensível. Consequentemente, eventuais erros associados a incertezas na medição da temperatura da interface sólida são evitados; e, além disso, fica dispensado o uso de termopares ou sensores de temperatura, simplificando a execução dos ensaios.

Uma investigação sobre as possíveis causas dos desvios constatados entre os dados experimentais e valores teóricos previstos no modelo adotado reside no fato de que na convecção natural o escoamento do fluido ocorre a velocidades baixas. Assim, o escoamento do ar tende a ser afetado pela movimentação de pessoas no entorno da placa, alterando o valor do coeficiente de película. As flutuações constatadas na taxa de fusão do gelo, bem como o fato da taxa de transferência de calor experimental superar a taxa teórica, justificam as os desvios constatados no experimento.

O emprego de atividades experimentais como estratégia de ensino tem sido apontado como uma das formas mais eficientes para mitigar as dificuldades presentes no processo de ensino-aprendizagem. Além disso, as possibilidades de uso dessa estratégia de ensino abrangem desde atividades voltadas para a mera verificação de leis e teorias, até a análise de situações que privilegiam a reflexão sobre a natureza dos fenômenos físicos e conceitos abordados em aula $[16,17]$. O experimento aqui descrito, cuja análise dos resultados requer uma reflexão mais ampla sobre o processo de transferência de calor - tendo em vista 
Tabela 2: Valores calculados com base nas equações descritas no texto.

\begin{tabular}{|l|l|l|}
\hline$L=0,528 \mathrm{~m}$ & $\rho_{s}=1,291 \mathrm{~g} / \mathrm{L}$ & $\sigma=5,6.10^{-8} \mathrm{~W} /\left(\mathrm{m}^{2} . \mathrm{K}^{4}\right)$ \\
\hline$\beta=3,53.10^{-3} \mathrm{~K}^{-1}$ & $\rho_{v, \infty}=0,015 \mathrm{~g} / \mathrm{L}$ & $\varepsilon=0,95$ \\
\hline$\gamma_{a r}=1,45.10^{-5} \mathrm{~m}^{2} / \mathrm{s}$ & $\rho_{a, \infty}=1,195 \mathrm{~g} / \mathrm{L}$ & $\boldsymbol{q}_{\mathbf{3}}=\mathbf{1 2 , 1 9} \mathbf{~ W}$ \\
\hline$\alpha_{a r}=2,05.10^{-5} \mathrm{~m}^{2} / \mathrm{s}$ & $\rho_{\infty}=1,210 \mathrm{~g} / \mathrm{L}$ & $\boldsymbol{q}_{\text {teorico }}=\mathbf{q}_{\mathbf{1}}+\mathbf{q}_{\mathbf{2}}+\mathbf{q}_{\mathbf{3}}$ \\
\hline$N_{g r}($ eq. 2$)=7,99.10^{7}$ & $D_{A B}=2,26.10^{-5} \mathrm{~m}^{2} / \mathrm{s}$ & $=\mathbf{3 4 , 8 3} \mathbf{W}$ \\
\hline$N_{n u}=51,2$ & $N_{g r}($ eq. 12$)=7,06.10^{-7}$ & $m_{1}=6,97 \mathrm{~g} / \mathrm{min}$ \\
\hline$k=2,49.10^{-2} \mathrm{~W} /(\mathrm{m} . \mathrm{K})$ & $N_{s h}=48,43$ & $m_{2}=6,69 \mathrm{~g} / \mathrm{min}$ \\
\hline$h=4,48 \mathrm{~W} /\left(\mathrm{m}^{2} . \mathrm{K}\right)$ & $h_{m}=3,84.10^{-3} \mathrm{~m} / \mathrm{s}$ & $L_{f}=334,4 \mathrm{~J} / \mathrm{g}$ \\
\hline$A=0,12 \mathrm{~m}^{2}$ & $m_{\text {cond. }}=4,6.10^{-3} \mathrm{~g} / \mathrm{s}$ & $\boldsymbol{q}_{\text {experimental }}=\mathbf{3 7 , 3 1} \mathbf{W}$ \\
\hline $\boldsymbol{q}_{\mathbf{1}}=\mathbf{1 1 , 2 4} \mathbf{W}$ & $L_{v a p}=2476 \mathrm{~J} / \mathrm{g}$ & Diferença $=\mathbf{7 2} \mathbf{1 2}$ \\
\hline$\rho_{v, s}=0,005 \mathrm{~g} / \mathrm{L}$ & $\boldsymbol{q}_{\mathbf{2}}=\mathbf{1 1 , 3 9} \mathbf{W}$ & \\
\hline$\rho_{a, s}=1,287 \mathrm{~g} / \mathrm{L}$ & & \\
\hline
\end{tabular}

que exige a quantificação das parcelas correspondentes a três mecanismos distintos - não se limita à verificação experimental de uma lei expressa através de uma equação padronizada. A simplicidade dos ensaios, cuja execução é realizada sem quaisquer equipamentos sofisticados ou de custo elevado, e dispensa o emprego de instrumentos de medição, à exceção de uma proveta, não constitui entrave para sua utilização como ferramenta pedagógica no estudo da transferência de calor. Na realidade, o fato da atividade prática consistir essencialmente em medidas de massa, bem como a concordância dos resultados experimentais como o modelo proposto, possibilita uma investigação qualitativa e quantitativa mais profunda sobre os mecanismos envolvidos no comportamento térmico de um sistema real.

A simplicidade dos ensaios realizados e do equipamento empregado são aspectos relevantes do experimento ora descrito, que viabilizam sua implementação em disciplinas que tratam da transferência de calor e massa. Tais características podem ser particularmente interessantes no caso de instituições de ensino que enfrentam dificuldades financeiras e burocráticas na aquisição de equipamentos destinados a aulas experimentais em seus cursos de graduação.

\section{Conclusões}

Um dos principais objetivos desse trabalho foi a elaboração de um equipamento simples para ser utilizado em um ensaio de fácil execução, de modo a comprovar a viabilidade da realização de aulas práticas como uma ferramenta pedagógica eficiente para o ensino de Fenômenos de Transporte. É possível afirmar que o experimento atingiu seus objetivos, tendo em vista a concordância dos dados experimentais com os valores teóricos previstos no balanço térmico formulado para o sistema físico proposto.

Além disso, a análise desenvolvida na elaboração do balanço térmico formulado para esse sistema possibilita ao aluno constatar a ocorrência de três mecanismos simultâneos de transferência de calor e massa, sendo todos eles relevantes no processo global de absorção de calor por uma placa de gelo. Finalmente, cabe destacar que o experimento ora descrito, embora simples e de fácil execução, envolve o estudo quantitativo de vários processos físicos vinculados a transferência de calor e massa, levando o aluno a se familiarizar com os valores numéricos desses parâmetros e com o emprego das equações associados a esses processos, a partir de um trabalho prático e realizado em grupo.

Os autores concluem que esse trabalho, pelo fato de apresentar um experimento didático simples, de fácil execução, cujos resultados experimentais são satisfatórios e cuja implementação em disciplinas de graduação não requer maiores esforços, pode contribuir para o ensino da transferência de calor e massa em cursos de graduação e ser utilizado como fonte de consulta para a elaboração de experimentos similares.

\section{Agradecimentos}

Os autores gostariam de agradecer à Fundação de Amparo à Pesquisa do Estado do Rio Grande do Sul (FAPERGS) pelo suporte financeiro a esse projeto.

\section{Referências}

[1] L.D.F. Marczak, I.C. Tessaro, N.S.M. Cardozo e K. Wada, in: XXXI COBENGE, Rio de Janeiro, 2003.

[2] P. Tamir, J Res Sci Teach 14, 311 (1977). 
[3] R. Moraes, Construtivismo e Ensino de Ciências: Reflexões Epistemológicas e Metodológicas (Ed. EIDUCRS, Porto Alegre, 2003), p. 195-208.

[4] E. Lüdke, A.G.R. Adornes, C.A. Gomes e R.B. Adornes, Rev. Bras. Ens. Fis. 35, 2 (2013).

[5] R.L. Garcia, D.A. Falcão, J.A. Della Giustina Neto, C. Pibernat e J.R. Zabadal, Revista Eletrônica Científica da UERGS 2, 54 (2016).

[6] A. Bejan, Transferência de Calor (Ed. Edgard Blucher, São Paulo, 1996), p. 570.

[7] F. Kreith, Princípios da Transmissão de Calor (Ed. Edgard Blucher, São Paulo, 1973).

[8] F.P. Incropera e D.P. de Witt, Transferência de Calor e Massa (Ed. LTC, Rio de Janeiro, 2008).

[9] J.P. Holman, Transferência de Calor (Ed. Mc GrawHill, São Paulo, 1983).

[10] R.B. Bird, W.E. Steward e E.N. Lightfoot, Fenômenos de Transporte (Ed. LTC, Rio de Janeiro, 2004).

[11] J.R. Welty, C.E. Wicks and R.E. Wilson, Fundamentals of Momentum, heat and Mass Transfer ( Ed. John Wiley \& Sons Inc., New York, 1984).

[12] Y.A. Çengel, Transferência de Calor e Massa: Uma Abordagem Prática (Ed. McGraw-Hill, São Paulo, 2009).

[13] J.F. Kreider and F. Kreith, Principles of Solar Energy Handbook (Ed. Hemisphere Publishing Co., New York, 1978).

[14] L.E. Sisson e D.R. Pitts, Fenômenos de Transporte (Ed. Guanabara Dois, Rio de Janeiro, 1996).

[15] M.S.T. Araújo e M.L.V.S. Abib, Rev. Bras. Ens. Fis. 25, 176 (2003).

[16] D. Hodson, Enseñanza de Las Ciências 12, 299 (1994).

[17] R.F. White, Int J Sci Educ 18, 761 (1996). 